Pacific Journal of Mathematic 


\title{
COMPLETION AND SEMICOMPLETION OF MOORE SPACES
}

\author{
JOHN WILLIAM GREEN
}

\begin{abstract}
Two properties are given which characterize those metacompact Moore spaces that are completable and two which characterize those which are semicompletable. The relationship among these properties in nonmetacompact spaces is investigated. Two new characterizations of Moore-closed spaces are given as is a characterization of developability. Some unification of technique is achieved among the various ways of completing or semicompleting a Moore space.
\end{abstract}

In 1962, O. H. Alzoobaee stated two properties $A$ and $B$ of developments and showed that every Moore space having a development with property (A) $B$ is (semicompletable) completable and every (semicomplete) complete Moore space has a development with property (A) $B$. In the present work, it is shown that every metacompact (semicompletable) completable Moore space has a development with property (A) $B$. Related properties $A^{\prime}$ and $B^{\prime}$ are investigated. It is shown that a Moore space is Moore-closed iff every strong development has property $A$ and iff every strong development has property $B$. Finally, a developable topological space is shown to have a sequence $\left\langle G_{n}\right\rangle$ of open covers such that for each point $p$ and open set $U$ containing $p$, there is an $n$ such that only one member of $G_{n}$ contains $p$ and it is a subset of $U$.

Complete Moore spaces were introduced in [7] and it was shown in [12] and [17] that a metrizable space is metrically topologically complete iff it is a complete Moore space. Semicompleteness (sometimes called Rudin completeness), a weaker property, was introduced in [14] and shown to be equivalent to completeness in metrizable spaces. Unlike the theories of metrizable or uniformizable spaces, there exist Moore spaces that cannot be embedded in any semicomplete Moore space [14], [9] and there exist semicomplete Moore spaces that cannot be completed [14]. The question naturally arises then of characterizing (semi-) completable Moore spaces. Whipple [15] has characterized completable Moore spaces in terms of Cauchy sequences and Creede [3] has what might be called an external characterization in terms of the Wallman compactification. An external characterization of semicompletability is given by J. N. Reed in [11]. Stronger completeness properties are investigated in [4] and [5]. The properties of Alzoobaee mentioned above were introduced in [1] and reported 
in [2] and are internal properties. The above mentioned characterization of developability has a bearing on the idea of $\theta$-base [16] and is motivated in part by [13].

1.2. Terminology. A development for a topological space $S$ is a sequence $G=G_{1}, G_{2}, \cdots$ of open covers of $S$ such that for each point $x$ and neighborhood $U$ of $x$, there is an $n$ such that st $\left(x, G_{n}\right) \subseteq U$. The development $G$ will be called monotonic if $G_{n} \supseteqq G_{n+1}$ for each $n$ and nested if in addition, $\bar{G}_{n+1}$ refines $G_{n}$. A Moore space is a regular Hausdorff developable space. A Moore space is (1) complete (2) semicomplete iff there is a monotonic development $G$ for $S$ such that if $M_{1}, M_{2}, \ldots$ is a nonincreasing sequence of closed point sets and $g_{1}, g_{2}, \ldots$ is a sequence such that for each $n, g_{n} \in G_{n}$, then (1) if $M_{n} \subseteq \bar{g}_{n}$ for each $n$, then $\cap M_{n} \neq \varnothing$, (2) if $\bar{g}_{n+1} \subseteq g_{n}$ for each $n$, then $\cap \bar{g}_{n} \neq \varnothing$. It is shown in [14] that if the closure symbol is omitted in (1) or in its first occurrence in (2), then the resulting statements are equivalent to those given. If $G$ is a monotonic development for a Moore space $S$ then $F=\left\{f_{n}: n \in N\right\}$ is (1) a $G$-filtersubbase, (2) a $G$-filterbase, (3) a $G$-nest [2] iff for each $n, f_{n} \in G_{n}$ and (1) $F$ is a filtersubbase (i.e., the intersection of each finite subcollection of $F$ is nonempty), (2) $f_{n} \supseteqq f_{n+1}$ for each $n$, (3) $f_{n} \supseteqq \bar{f}_{n+1}$ for each $n$, respectively. A filtersubbase $F$ intersects a set $M$ iff every set in $F$ intersects $M$ and two filterbases are said to intersect iff every set in one intersects every set in the other. If $G$ is a development for the Moore space $S$, then $G$ is said to have property $A^{\prime}, A, B^{\prime}, B$ according as $G$ is monotonic and $\left(A^{\prime}\right)$ if $U$ and $V$ are members of $G_{1}$ and $U$ is a proper subset of $V$ and $F$ is a $G$-nest intersecting $U$, then there is a positive integer $n$ such that if $W \in G_{n}$ and $F$ intersects $W$, then $W \subseteq V$.

(A) $A$ is obtained from $A^{\prime}$ by replacing the phrase " $U$ is a proper subset of $V$ " with the phrase " $\bar{U} \subseteq V$ ".

(B') If $U \in G_{1}$ and $F$ is a $G$-filtersubbase some member of which is a proper subset of $U$, then there is a positive integer $n$ such that if $W \in G_{n}$ and $F$ intersects $W$, then $\bar{W} \cong U$.

(B) $B$ is obtained from $B^{\prime}$ by replacing the phrase "some member of which is a proper subset of $U$ " with the phrase "the closure of some member of which is included in $U$ ".

It should be observed that if $G$ has property $A^{\prime}$ or $B^{\prime}$, then $G_{1}$ is a nested basis for the topology of $S$; that is, if $U$ and $V$ belong to $G_{1}$ and $U$ is a proper subset of $V$, then $\bar{U} \subseteq V$. The argument for Theorem 9 of [5] shows that every metacompact Moore space has a nested basis. In 3.3 a nonmetacompact Moore space having a nested basis is indicated.

1.3. Preliminaries. If $G$ is a nested development for the Moore 
space $S$ and $\alpha$ is a sequence of points and $U$ is an open set, then $\alpha$ is said to be $G$-interior to $U$ [15] iff (1) $\alpha$ is $G$-Cauchy (for each $n$, some member of $G_{n}$ contains a final segment of $\alpha$ ) and (2) for some $n$, every member of $G_{n}$ containing a final segment of $\alpha$ is a subset of $U$. The open set $U$ is $G$-interior to the open set $V$ iff every $G$ Cauchy sequence having a final segment in $U$ is $G$-interior to $V$. In [15] it is proved that a Moore space $S$ is completable iff there is a nested development $G$ for $S$ such that if $\alpha$ is a $G$-interior sequence of the open set $U$, then for each $n$ there is a $G$-interior subset of $U$ belonging to $G_{n}$ and containing a final segment of $\alpha$. A developmet with this property will be said to have Whipple's property. It is easily demonstrated that if $G$ is a nested development with Whipple's property, then (1) for each $G$-Cauchy sequence $\alpha$, there is a $G$-nest $G(\alpha)=\left\{g_{n}(\alpha): n \in N\right\}$ such that $g_{n+1}(\alpha)$ is $G$-interior to $g_{n}(\alpha)$ and contains a final segment of $\alpha$, (2) $G(\alpha)$ intersects $G(\beta)$ iff $G(\alpha)$ and $G(\beta)$ are equivalent filterbases. Finally, some unification of technique among [15], [1], [2], [11], and the more general [8] (see [6] for additional references and results using the technique of [8]) is obtained by observing that if $G$ is a nested development with Whipple's property, $H$ is a collection of free nonintersecting $G$-nests such that every free $G$-nest intersects a member of $H$ and for each open set $U$ in $S, U_{H}^{*}=$ $U \cup\{F \in H: U$ includes a member of $F\}$, then $S_{H}^{*}$, with $\left\{U_{H}^{*}: U\right.$ open in $S\}$ as a basis, is a complete Moore space having $S$ as a dense subspace and $G_{H}^{*}$, defined in the obvious fashion, is a complete monotonic development for $S_{H}^{*}$.

2. Completion and semicompletion in arbitrary Moore spaces.

LEMMA 2.1. If $S$ is a complete (semicomplete) Moore space and $G$ is a monotonic development for $S$, then there is a complete (semicomplete) nested development $H$ for $S$ such that for each $n, H_{n} \subseteq G_{n}$.

The argument for the complete case is entirely trivial and for the semicomplete case is a straightforward application of Rudin's lemma ([14], Theorem 2).

LEMMA 2.2. The property that every $x$ of the Moore space $S$ has a neighborhood having no point other than $x$ as a limit point implies that $S$ is semicomplete but not that $S$ is complete.

Proof. Under the stated hypothesis, there is a nested development $G$ for $S$ such that if $U \in G_{1}$ then either $U$ is singleton or for some point $x$ of $U, x$ is the only limit point of $U$. Suppose for each $n$, $g_{n+1} \subseteq g_{n} \in G_{n}$. If for some $n, g_{n}$ is singleton, then $\cap g_{i}=g_{n}$. Suppose 
for each $n, g_{n}$ has only one limit point, $x_{n}$. If for some $i$ and $j, x_{i} \neq$ $x_{j}$, then for each $n>i+j$, no nonsingleton member of $G_{n}$ is a subset of $g_{i} \cap g_{j}$, contrary to supposition. Thus, $x_{n}=x_{1}$ for each $n$ and $\cap g_{n}=\left\{x_{1}\right\}$. Thus, $G$ is a semicomplete development for $S$.

Theorem 9 of [14] provides an example of a semicomplete Moore space that is not complete. If this example is modified by requiring each point above the $x$-axis to be open, then the resulting space satisfies the hypotheses of this lemma and Rudin's argument, with only the obvious modifications, shows the space is not complete.

THEOREM 2.3. If $D$ is an open (semi-) complete subspace of the (semi-) completable space $S$, there is a (semi-) completion of $S$ having $D$ as an open subset.

Proof (Complete Case). Let $S^{\prime}$ denote a completion of $S$ and for each subset $A$ of $S$, let $\bar{A}$ denote the closure of $A$ in $S^{\prime}$. Then $D \cup \overline{S-D}$ is a completion of $S$ having $D$ as an open subset. For clearly $D$ is open in this space. Let $\mathrm{Cl} A=$ closure in $D \cup \overline{S-D}$ of A.

Since $\overline{S-D}$ is closed in $S^{\prime}$, there exist sets $U_{1}, U_{2}, \cdots$ open in $S^{\prime}$ such that $\overline{S-D}=\bigcap_{i} U_{i}$. Let $F=F_{1}, F_{2}, \cdots$ denote a complete development for $D$ such that for each $n$ and each $f \in F_{n}, \mathrm{Cl}_{D} f=\mathrm{Cl} f$.

Let $G=G_{1}, G_{2}, \ldots$ denote a complete development for $S^{\prime}$. For each $i$, let

$$
H_{i}=\left\{g \cap(D \cup \overline{S-D}): g \in G_{i} \text { and } \bar{g} \subseteq U_{i}\right\} .
$$

Finally, for each $i$, let $K_{i}=F_{i} \cup H_{i}$. Clearly, $K=K_{1}, K_{2}, \cdots$ is a development for $D \cup \overline{S-D}$. Suppose $M_{1}, M_{2}, \ldots$ is a nonincreasing sequence of closed point sets in $D \cup \overline{S-D}$ and for each $i, M_{i} \subseteq \mathrm{Cl} k_{i}$ and $k_{i} \in K_{i}$.

Case 1. For infinitely many $i, k_{i} \in H_{i}$. Then $\bigcap_{i} \bar{M}_{i} \subseteq \bigcap_{i} \bar{k}_{i}$ and since $H_{1}, H_{2}, \ldots$ is a complete development for $S^{\prime}, \bigcap_{i} \bar{M}_{i} \neq \varnothing$. Since $k_{i} \in H_{i}$ implies $\bar{k}_{i} \subseteq U_{i}$, it follows that $\bigcap_{i} \bar{M}_{i} \subseteq \bigcap_{i} U_{i}=\overline{S-D}$. Hence, $\bigcap_{i} \bar{M}_{i}=\bigcap_{i} M_{i} \neq \varnothing$.

Case 2. For infinitely many $i, k_{i} \in F_{i}$. Then for each such $i$, Cl $M_{i} \subseteq \mathrm{Cl} k_{i} \leqq \mathrm{Cl}_{D} k_{i}$, so $M_{i}$ is closed in $D$. The completeness of $F$ implies $\bigcap_{i}$ CL $M_{i}=\bigcap M_{i} \neq \varnothing$.

2.4. Acknowledgment. The proofs of 2.3 and 2.5 and the last paragraph of the proof of 4.1 which appear here are considerably simpler than the author's original arguments and are due to the referee. 
THEOREM 2.5. If $M$ is a closed (semi-)complete subspace of the (semi-)completable Moore space $S$, there is a (semi-)completion of $S$ having $M$ as a closed subset.

Proof (Complete Case). Suppose $M$ is a closed, complete subspace of $S$ and $S^{\prime}$ is a completion of $S$. There is a set $V$ open in $S^{\prime}$ such that $V \cap S=S-M$. Now $\mathrm{Cl}_{S^{\prime}} M$ is a complete space and since $M$ is also complete, $M$ is a $G_{o}$ in $S^{\prime}$. Let $U_{1}, U_{2}, \ldots$ denote sets open in $S^{\prime}$ such that $\bigcap_{i} U_{i} \cap \mathrm{Cl}_{S^{\prime}} M=M$. Then $M$ is closed in $V \cup \bigcap_{i} U_{i}$ which is a $G_{\delta}$ in $S^{\prime}$ and hence complete.

Corollary 2.6. If $M$ is a subspace of the (semi-)complete Moore space $S$, then in order that there exists a (semi-)completion $T$ of $S$ such that $\mathrm{Cl}_{T} M=\mathrm{Cl}_{S} M$, it is necessary and sufficient that $\mathrm{Cl}_{S} M$ be (semi-)complete.

LEMMA 2.7. If $S$ is a Moore space for which at least one development has property $B^{\prime}$, then $S$ is completable.

Proof. There is a nested development $G$ for $S$ having property $B^{\prime}$. Suppose $\alpha$ is a $G$-interior sequence of the open set $D$, so that for some $n$, every member of $G_{n}$ containing a final segment of $\alpha$ is a subset of $D$. If for some point $x,\{x\}$ is open and contains a final segment of $\alpha$, then $\{x\} \in G_{i}$ for every $i$ and is $G$-interior to $D$. Suppose there is no singleton open set containing a final segment of $\alpha$. There exist $f_{1} \in G_{n}$ and $f_{2}$ in $G_{n+1}$ such that $f_{2}$ is a proper subset of $f_{1}$ and contains a final segment of $\alpha$. Suppose $\beta$ is a $G$-Cauchy sequence having a final segment in $f_{2}$. Suppose further that for each $m$, there exists $t_{m} \in G_{m}$ and containing a final segment of $\beta$ and intersecting $S-D$. Let $f_{i}=t_{i}$ for $i>2$ and $F=\left\{f_{i}: i \in N\right\} . \quad F$ is a $G$-filtersubbase containing $f_{2}$ and $f_{2}$ is a proper subset of $f_{1}$. Since $G$ has property $B^{\prime}$, there exists $m>2$ such that if $W \in G_{m}$ and $F$ intersects $W$, then $\bar{W} \subseteq f_{1}$. But $f_{m} \in G_{m}$ and $F$ intersects $f_{m}$ and $f_{m}$ intersects $S-f_{1}$. This is a contradiction. Therefore, $\beta$ is $G$-interior to $f_{1}$. It follows that $G$ has Whipple's property and $S$ is completable.

THEOREM 2.8. If some development for the Moore space $S$ has property $B^{\prime}$, then some development for $S$ has property $B$.

Proof. There exists a monotonic development $G$ for $S$ having property $B^{\prime}$. Let $M=\{x \in S$ : there is a neighborhood of $x$ having no point other than $x$ as a limit point\}. $M$ is an open semicomplete 2.2 subspace of a completable 2.7 space. By Theorem 5 of [14], $M$ is complete. Let $R=\left\{U \in G_{1}: U \cap(S-M) \neq \varnothing\right.$ and $U$ is closed $\}$. For 
each $U$ in $R$, let $x_{U}$ and $y_{U}$ denote distinct limit points of $U$ and let $U_{x}=U-\left\{x_{U}\right\}$ and $U_{y}=U-\left\{y_{U}\right\}$. Let $C_{n}=\left\{U \in G_{n}: U\right.$ is singleton or $U$ has only one limit point and is closed $\}. C$ is a monotonic development for the subspace $M$. With the aid of 2.1 it may be seen that there exists a complete monotonic development $D$ for $M$ such that (1) $D_{n} \subseteq C_{n}$, (2) every nonsingleton member of $D_{n+1}$ is a proper subset of some member of $D_{n}$. Since $\bar{D}_{n+1}$ refines $D_{n}$, it follows that $D$ has property $B$ for the subspace $M$ and since $D_{n} \subseteq C_{n}, D$ also has property $B^{\prime}$. Let $H_{n}=D_{n} \cup\left\{U \in G_{n}-R: U \cap(S-M) \neq \varnothing\right\} \cup\left\{U_{t}\right.$ : $U \in R \cap G_{n}$ and $t$ is $x$ or $\left.y\right\}$. There is a nested development $K$ for $S$ such that for each $n, K_{n} \subseteq H_{n+1}$.

Notation. If $U \in K_{1} \cap G_{1}$, let $U^{\prime}=U$. If $U \in K_{1}-G_{1}$, let $U^{\prime}$ denote a set in $R$ such that $U^{\prime}-\left\{t_{U}\right\}=U$ for some $t \in\{x, y\}$. If $F \subseteq K_{1}$, let $F^{\prime}=\left\{f^{\prime}: f \in F\right\}$.

Suppose $U$ and $V$ belong to $K_{1}$ and not both are subsets of $M$ and $\bar{U} \subseteq V . \quad V$ is not closed since no member of $K_{1}-D_{1}$ is a subset of any member of $D_{1}$. Since $U^{\prime}=\bar{U} \cong V$, it follows that $U^{\prime}$ is a proper subset of $V$ and of $V^{\prime}$.

Suppose $V \in K_{1}-D_{1}$ and $F$ is a $K$-filtersubbase the closure of some member $f$ of which is a subset of $V$. As shown above, $f^{\prime}$ is a proper subset of $V^{\prime}$. Thus, there is an integer $n$ such that if $W \in G_{n}$ and $F^{\prime}$ intersects $W$, then $\bar{W} \subseteq V^{\prime}$. Suppose for each $i \geqq n$ there is a set $W_{i} \in K_{i}$ such that $F$ intersects $W_{i}$ and $\bar{W}_{i}$ is not a subset of $V$. $F^{\prime}$ intersects $W_{i}^{\prime}$, so $\bar{W}_{i}^{\prime}=\bar{W}_{i} \subseteq V^{\prime}$. There is a point $p$ such that $V^{\prime}-\{p\}=V$ and it follows that $p \in \bar{W}_{i}$ for each $i$. Since $\bar{K}_{i+1}$ refines $K_{i}$, there exists $Z_{i} \in K_{\imath}$ containing $\bar{W}_{i}$ and thus containing $p$. It follows that $Z=\left\{Z_{2}: i>n-2\right\}$ is a local base at $p$ and contains a $K$-nest. $\bar{f} \leqq V$ and $p \notin V$, so $p \notin \bar{f}$ and there is an $m>n$ such that $Z_{m}^{\prime} \cap f=\varnothing$. There exists $j>m$ such that $Z_{j}^{\prime}$ either is $\{p\}$ or is a proper subset of $Z_{m}^{\prime}$.

Case 1. $Z_{j}^{\prime}$ is a proper subset of $Z_{m}^{\prime}$. Since $G$ has property $B^{\prime}$, there is an $i>j$ such that if $W \in G_{i}$ and $Z$ intersects $W$, then $\bar{W} \cong$ $Z_{m}^{\prime}$. In particular, $\bar{f}_{i} \subseteq Z_{m}^{\prime}$. But $f \cap Z_{m}^{\prime}=\varnothing$ and $f \cap f_{i} \neq \varnothing$. This is a contradiction.

Case 2. $Z_{j}^{\prime}=\{p\}$. There is an $i>j$ such that $\operatorname{st}\left(p, G_{i}\right)=\{p\}$. Since $F$ intersects $\{p\}$, it is necessary that $p \in \bar{f}$. This is again a contradiction. Therefore, there is an $i>n$ such that if $W \in K_{i}$ and $F$ intersects $W$, then $\bar{W} \subseteq V$.

Suppose $V \in D_{2}$ and $F$ is a $K$-filtersubbase such that for some $f$ in $F, \bar{f} \subseteq V$. There exists $U$ in $D_{1}$ such that either $U$ is singleton 
and is $V$ or $U$ has $V$ as a proper subset.

Case 1. $V$ is a proper subset of $U . \quad U$ and $V$ belong to $G_{1}$, so there is an $n$ such that if $F^{\prime}$ intersects $W^{\prime}$ and $W^{\prime} \in G_{n}$, then $\bar{W}^{\prime} \cong$ $U$. If $W \in K_{n}$ and $F$ intersects $W$, then $\bar{W} \subseteq U$, so that $W \in D_{1}$. Thus, $F$ contains a $D$-filtersubbase having $f$ as a member. Since $D$ has property $B$, there is an $i>n$ such that if $W \in D_{i}$ and $F$ intersects $W$, then $\bar{W} \subseteq V$.

Case 2. $U=\{x\}$ for some point $x$ of $M$. For some $i$, st $\left(x, G_{i}\right)=$ $\{x\}$. If $W \in G_{i}$ and $x \in \bar{W}$, then $W=\{x\}$. Thus, $f_{i}=\{x\}$ and if $W \in$ $G_{i}$ and is intersected by $F$, then $W=\{x\} \subseteq U=V$.

Therefore, $K$ has property $B$.

THEOREM 2.9. If some development for the Moore space $S$ has property $A^{\prime}$, then some development for $S$ has property $A$.

This may be established by a minor alteration of the proof of Theorem 2.8 .

THEOREM 2.10. In order that a Moore space $S$ be (semi-)completable, it is sufficient that some development for $S$ have property $\left(A^{\prime}\right) B^{\prime}$.

This follows from Theorems 2.8 and 2.9 above and 3.7 and 4.4 of [2].

THEOREM 2.11. Every (semi-)complete Moore space having a nested basis has a development with property $\left(A^{\prime}\right) B^{\prime}$.

Proof. Suppose $D$ is a nested basis for the (semi-)complete Moore space $S$. There exists a nested (semi-)complete development $G$ for $S$ such that $G_{1} \subseteq D$. Such a development must have property (A) $B$ and from this and the fact that $G_{1}$ is a nested basis, $\left(A^{\prime}\right) B^{\prime}$ follows.

2.12. It is easily demonstrated that $B^{\prime}$ implies $A^{\prime}$ for any monotonic development. (This parallels Theorem 4.2 of [2].) It may be shown along the lines of the argument for Theorem 10 of [5] that if a Moore space has a completion having a nested basis of regular open sets, then some development for $S$ has property $B^{\prime}$. It may also be of interest that an argument like that for Theorem 9 of [5] may be used to obtain a characterization of metrizable spaces related to property $C$ of [2] in the same way that $B^{\prime}$ is related to $B$. For the next result, a Moore-closed space [5] is a Moore space that is a 
closed subspace of every Moore space containing it. A development $G$ is strong [15] iff it is monotonic and for each point $x$ and neighborhood $U$ of $x$, there is a positive integer $n$ such that if $W \in G_{n}$ and $x \in \bar{W}$, then $W \cong U$. It is easily verified that a (semi-)complete monotonic development for a Moore space $S$ has property (A) $B$ iff it is strong, and that every nested development is strong (but not conversely). $1 \rightarrow 3$ of the next result provides a striking contrast to Theorem 3 of [5] since it follows from hypothesis 3 that every strong development is complete but it does not follow that $S$ is compact, while the property that every monotonic development is complete characterizes compactness. From a private communication with G. M. Reed, the author has learned that Reed, independently and somewhat earlier than the date of 2.13, characterized Moore-closed spaces as the Moore spaces in which every strong development is complete.

THEOREM 2.13. If $S$ is a Moore space, the following are equivalent:

(1) $S$ is Moore-closed.

(2) Every strong development for $S$ has property $A$.

(3) Every strong development for $S$ has property $B$.

Proof. (1 $\rightarrow 3)$. Suppose $S$ is Moore-closed, $G$ is a strong development for $S, U \in G_{1}$ and $F$ is a $G$-filtersubbase the closure of some member of which is a subset of $U . \quad\left\{\bigcap_{i \leqq n} f_{i}: n \in N\right\}$ is a countable open filterbase and by the argument for Theorem 2 of [5] $S$ is feebly compact, so there is a point $p$ in $\bigcap_{n} \bar{f}_{n}$. Suppose $D$ is an open set containing $p$. Since $G$ is strong, there is a positive integer $n$ such that if $k \geqq n$, then $\bar{f}_{k} \subseteq D$. Thus, $F$ converges to $p$ and if $W$ is a set intersected by $F$, then $p \in \bar{W}$. Therefore, there is an $m$ such that if $W \in G_{m}$ and $F$ intersects $W$, then $\bar{W} \subseteq U$.

$(3 \rightarrow 2)$ is a consequence of Theorem 4.2 of [2].

$(2 \rightarrow 1)$. Suppose $S$ is a Moore space that is not Moore-closed. There exist a Moore space $S^{\prime}$ having $S$ as a dense subspace, a point $p$ and a strong development $G^{\prime}$ for $S^{\prime}$ such that $S^{\prime}-S=\{p\}$ and $p$ is a limit point of $S$. There exist sequences $\left\{x_{\imath}\right\}$ and $\left\{y_{i}\right\}$ of points of $S$ and $G^{\prime}$-nests $\left\{R_{\imath}\right\},\left\{T_{n i}\right\}_{i}$, and $\left\{Q_{n_{2}}\right\}_{i}$ for each $r$ such that (a) $x$ and $y$ converge to $p$, (b) $x_{n} \in T_{n i+1} \subseteq \bar{T}_{n i+1} \subseteq T_{n i} \subseteq R_{n}-\bar{R}_{n+1}$, (c) $y \in$ $Q_{n+1} \subseteq \bar{Q}_{n i+1} \subseteq Q_{n i} \subseteq R_{n}-\bar{R}_{n+1}$, (d) $p \in R_{n}$, (e) $T_{n 1} \cap Q_{n 1}=\varnothing$ for each $n$. Let $U=R_{2}-\mathbf{U}_{n} \bar{T}_{n 1}-\{p\}, V=R_{1}-\mathbf{U}_{n} \bar{T}_{n 2}-\{p\}, f_{n}=\mathbf{U}_{i \geqq n} T_{\imath i} \cup$ $Q_{\imath i}$ and $F=\left\{f_{i}: i>1\right\}$. For each $n$, let $G_{n}=\left\{g \in G_{n}^{\prime}: p \in \bar{g}\right.$ and either $g \cap f_{n+1}=\varnothing$ or $g \subseteq T_{k n}$ or of $Q_{k n}$ for some $\left.k \geqq n\right\}$. For $n>1$, let $H_{n}=G_{n} \cup\left\{f_{k}: k \geqq n\right\}$ and let $H_{1}=H_{2} \cup\{U, V\}$. $H$ is a strong development for $S, \bar{U} \subseteq V$ (in $S$ ), $U$ and $V$ belong to $G_{1}$ and $F$ is an $H$-nest intersecting both $U$ and $S-V$. Thus, $H$ does not have property $A$. 
2.14. "Strong" cannot be omitted from (2) of 2.13. For consider the space $\Delta$ of Example 9, page 66 of [7]. Let $\alpha$ and $\beta$ be distinct members of $Z$ and suppose "region" means what is intended in the cited example or one of these sets $W_{n}=\{x$ : for some $k \geqq n, x$ is the $k$ th term of $\alpha$ or of $\beta$. These new regions can be used to construct a monotonic development $G$ for $\Delta$ that is not strong. But $\Delta$ is Mooreclosed (see the section headed "Examples" in [5]) so by Theorem 2 of [5] $G$ is semicomplete. From 2.12, $G$ cannot have property $A$.

\section{Metacompact spaces.}

THEOREM 3.1. If $S$ is a metacompact Moore space, the following are equivalent:

(1) $S$ is completable.

(2) Some development for $S$ has property $B$.

(3) Some development for $S$ has property $B^{\prime}$.

Proof. In view of Theorem 2.8 above and 4.4 of [2], all that remains to be shown is that $(1 \rightarrow 3)$. Under the hypothesis of $(1)$, there is a nested development $K$ for $S$ having Whipple's property. There exists a sequence $B_{1}, B_{2}, \ldots$ of point-finite open collections covering $S$ minimally such that for each $n, B_{n+1}$ refines $B_{n}$ and $K_{n+1}$. Let $C_{1}=B_{1}$ and for each $n$, let $C_{n+1}$ denote the collection of all members $U$ of $B_{n+1}$ such that for every $V$ in $\bigcup_{i \leqq n} C_{i}$, either $U \cap(S-$ $V) \neq \varnothing$ or $U$ is $K$-interior to $V$.

Suppose for some $m, \bigcup_{i \geqq m} C_{i}$ does not cover $S$ and $p$ is a point not covered by this collection. Let $U_{1}, U_{2}, \ldots, U_{n}$ denote the members of $\bigcup_{i<m} C_{i}$ containing $p$ and let $U=\bigcap_{i \leqq n} U_{i}$. The constant sequence $p$ is $K$-interior to $U$. By Whipple's property, there is a set $R$ in $K_{m}$ containing $p$ which is $K$-interior to $U$. There is a $k \geqq m$ such that st $\left(p, G_{k}\right) \subseteq R$. There is a set $V$ in $B_{k}$ containing $p$. $V \subseteq R$, so $V$ is $K$-interior to $U$. Therefore, either $V \in C_{k}$ or there is a set in $\bigcup_{m<i<k} C_{i}$ containing $p$. In either case a contradiction is obtained.

Let $H_{1}=\bigcup_{i} C_{i}$ and for each $n$, let $H_{n+1}=\left\{U \in \bigcup_{i>n} C_{i}: U\right.$ is $K$ interior to some set in $\left.H_{n}\right\} . \quad H$ is a nested development for $S$. For clearly $H_{1}$ covers $S$ and suppose $p$ is a point and $n$ a positive integer such that $H_{n}$ covers $S$. There exists $U$ in $H_{n}$ containing $p$ and $m>n$ such that st $\left(p, K_{m}\right) \subseteq U$. There is a set $V$ in $\bigcup_{i \geqq m} C_{i}$ containing $p$. $V \cap(S-U)=\varnothing$, so $V$ is $K$-interior to $U$. $V \in H_{m} \subseteq H_{n+1}$ and it follows that $H_{n+1}$ covers $S$. That $H$ is nested follows from the fact that every member of $H_{n+1}$ is $K$-interior to some member of $H_{n}$.

(i) If $U$ and $V$ are distinct members of $H_{1}$ and $U \subset V$, then $U$ is $K$-interior to $V$. For $V \in C_{n}$ for some $n$ and $U \in C_{k}$ for some $k$. If $k \leqq n$, then there is a set $W$ in $B_{k}$ containing $V$. But $U \subset V \subseteq W$ 
and $U$ and $W$ are thus distinct members of $B_{k}$. This contradicts the fact that $B_{k}$ covers $S$ minimally. Therefore, $k>n$ and by construction of $C_{k}, U$ is $K$-interior to $V$.

(ii) $H$ has Whipple's property. This may be shown along the lines of the argument for Theorem 3 of [15].

There exists a collection $T^{\prime \prime}$ such that (1) if $F \in T^{\prime}$, then $F=H(\alpha)$ for some $H$-Cauchy sequence $\alpha$ (cf. 1.3), (2) if $\alpha$ is an $H$-Cauchy sequence, then $H(\alpha)$ intersects some member of $T^{\prime \prime}$, (3) no two members of $T^{\prime \prime}$ intersect. Let $T$ denote the collection of all free filterbases in $T^{\prime}$. As observed in 1.3, $S_{T}^{*}$ is a completion of $S$ and $H_{T}^{*}$ is a complete monotonic development for $S_{T}^{*}$.

(iii) $H_{T}^{*}$ is nested and has property $B$. For if $U^{*} \in H_{n+1}^{*}$ (the subscript $T$ will be dropped here), there is a set $V$ in $H_{n}$ such that $U$ is $K$-interior to $V$. Then $\bar{U} \cong V$ (in $S$ ) and if for some $H(\alpha)$ in $T, H(\alpha)$ intersects $U$ (i.e., $H(\alpha) \in \mathrm{Cl} U^{*}$ (in $S^{*}$ )), then $H(\alpha)$ is equivalent to $H(\beta)$ for some $H$-Cauchy sequence $\beta$ having a final segment in $U$ and therefore, $H(\alpha) \in V^{*}$. So, $\mathrm{Cl}_{s^{*}} U^{*} \subseteq V^{*}$. So $H^{*}$ is nested and complete and this suffices to show $H^{*}$ has property $B$.

(iv) $H$ has property $B^{\prime}$. For suppose $U \in H_{1}$ and $F$ is an $H$ filtersubbase such that some member $V$ of $F$ is a proper subset of $U$. By (i), $V$ is $K$-interior, hence $H$-interior, to $U$. By the argument for (iii), $\mathrm{Cl}_{S^{*}} V^{*} \leqq U^{*}$. Since $H^{*}$ has property $B$, there is a positive integer $n$ such that if $W^{*} \in H_{n}^{*}$ and $F^{*}$ intersects $W^{*}$, then $\mathrm{Cl}_{S^{*}} W^{*} \leqq$ $U^{*}$. If $W \in H_{n}$ and $F$ intersects $W$, then $F^{*}$ intersects $W^{*}$. Therefore, $H$ has property $B^{\prime}$.

3.2. There is a metacompact Moore space that is not semicompletable. In [10], G. M. Reed makes the very fine observation that the first nonsemicompletable Moore space constructed in [9] is metacompact.

3.3. There exist nonmetacompact Moore spaces having property $B^{\prime}$. The space $\Delta$ cited in 2.14 is not metacompact and its topology has a basis of sets both open and closed. Now every topology with such a basis has a nested basis and so, by Theorem 2.11, it has property $B^{\prime}$. By the same reasoning, the example given in the proof of Lemma 2.2 has property $\mathrm{A}^{\prime}$.

THEOREM 3.4. If $S$ is a metacompact Moore space, the following are equivalent:

(1) $S$ is semicompletable.

(2) Some development for $S$ has property $A$.

(3) Some development for $S$ has property $A^{\prime}$.

Proof $(1 \rightarrow 3)$. (This again is all that need be shown.) Suppose 
$S^{\prime}$ is a semicompletion of the metacompact Moore space $S, G^{\prime}$ is a nested semicomplete development for $S^{\prime}$ and $G_{n}=\operatorname{Tr}_{S}\left(G_{n}^{\prime}\right) . \quad G$ is a monotonic development for $S$. By Rudin's lemma, there exist $H_{n} \subseteq$ $G_{n}$ and $H_{n}^{\prime} \subseteq G_{n}^{\prime}$ such that (a) $H_{n}$ and $H_{n}^{\prime}$ cover $S$ and $H_{n} \supseteqq H_{n+1}$ and $H_{n}^{\prime} \supseteqq H_{n+1}^{\prime}$, (b) if $h_{n} \in H_{n}$ and $h_{n} \supseteqq h_{n+1}$, then there exists an increasing sequence $\left\{p_{n}\right\}$ of positive integers and a sequence $\left\{h_{n}^{\prime}\right\}$ of sets such that $h_{n}^{\prime} \in H_{n}^{\prime}$ and contains $\mathrm{Cl}\left(h_{n}^{\prime} \cup h_{p_{n}}\right)$, where $\bar{M}$ and $\mathrm{Cl} M$ mean the closure of $M$ in $S$ and $S^{\prime}$, respectively. There exists a sequence $B_{1}$, $B_{2}, \cdots$ of point-finite open collections covering $S$ minimally such that $B_{1}$ refines $H_{1}$ and $\bar{B}_{n+1}$ refines $B_{n}$ and $H_{n+1}$. Let $C_{1}=B_{1}$ and for each $n$, let $C_{n+1}$ denote the collection of all members $U$ of $B_{n+1}$ such that for each $V$ in $\bigcup_{i \leqq n} C_{i}$, either (1) $U \cap(S-V) \neq \varnothing$ or (2) $\bar{U} \leqq V$ and if $F$ is an $H$-nest intersecting $U$ there is an $i$ such that if $W \in H_{i}$ and is intersected by $F$, then $W \subseteq V$. Finally, let $I_{n}=\bigcup_{i \geqq n} C_{i}$.

Suppose for some $m, I_{m}$ does not cover $S$ and $p$ is a point not covered by this collection. Let $V_{1}, V_{2}, \cdots, V_{n}$ denote the members of $\mathrm{U}_{i \leqq m} C_{i}$ containing $p$. There is a set $V^{\prime}$ in $H_{1}^{\prime}$ containing $p$ such that $V=V^{\prime} \cap S \leqq \bigcap_{i \leqq n} V_{i}$. There is a set $W^{\prime}$ in $H_{1}^{\prime}$ such that $p \in W^{\prime}$ and $\mathrm{Cl} W^{\prime} \subseteq V^{\prime}$. Let $W=W^{\prime} \cap S$, so $\bar{W} \cong V$. There is a set $R$ in $\bigcup_{i \geqq m} B_{i}$ containing $p$ such that $\mathrm{Cl} R \subseteq W^{\prime}$. Thus, $\bar{R} \subseteq V$. Suppose $F$ is an $H$-nest intersecting $R$ and $F^{\prime \prime}$ is an $H^{\prime}$-nest generated by $F$ as in (b). $F^{\prime}$ intersects $W^{\prime}$ and $\mathrm{Cl} W^{\prime} \subseteq V^{\prime}$. Since $H_{n}^{\prime} \subseteq G_{n}^{\prime}$ and $G^{\prime}$ has property $A$ for $S^{\prime}$, it follows that there exists an integer $q$ such that if $D^{\prime} \in G_{q}^{\prime}$ and $F^{\prime}$ intersects $D^{\prime}$, then $D^{\prime} \subseteq V^{\prime}$. Suppose $D \in H_{q}$ and $F$ intersects $D$. By construction, $D=D^{\prime} \cap S$ for some $D^{\prime} \in G_{q}^{\prime}$. Since $F$ intersects $D, F^{\prime}$ intersects $D^{\prime}$ and $D^{\prime} \subseteq V^{\prime}$. Thus, $D \subseteq V$. It follows that either $R \in I_{m}$ or there is some other set in $I_{m}$ containing $p$. This is a contradiction. Therefore, $I_{m}$ covers $S$ for each $m$.

Thus, $I$ is a monotonic development for $S$ such that if $U$ and $V$ belong to $I_{1}$ and $U$ is a proper subset of $V$ and $F$ is an $H$-nest intersecting $U$, then there is a positive integer $n$ such that if $W \in H_{n}$ and $F$ intersects $W$, then $W \cong V$. Rudin's lemma may be applied again resulting in statements $a^{\prime}$ and $b^{\prime}$ obtained from $a$ and $b$ by replacing $G^{\prime}$ with $H, G$ with $I, H$ with $J$, and $H^{\prime}$ with $J^{\prime}$ and in which closure always means with respect to $S$.

$J$ is a monotonic development for $S$ having property $A^{\prime}$. For suppose $U$ and $V$ belong to $J_{1}, U$ is a proper subset of $V$ and $F$ is a $J$-nest intersecting $U . \quad U$ and $V$ belong to $I_{1}$ and by $\left(b^{\prime}\right)$ there is an $H$-nest $F^{\prime \prime}$ generated by $F . \quad F^{\prime}$ intersects $U$, so there is a positive integer $n$ such that if $W \in H_{n}$ and $F^{\prime \prime}$ intersects $W$, then $W \subseteq V$. Suppose $W \in J_{n}$ and $F$ intersects $W$. By construction, there is a set $Z$ in $H_{n}$ including $W . \quad F^{\prime \prime}$ intersects $Z$ and $Z \subseteq V$, so $W \subseteq V$.

4. Developable spaces. 
THEOREM 4.1. The topological space $S$ is developable iff there is a sequence $G_{1}, G_{2}, \ldots$ of open covers of $S$ such that for each point $p$ and neighborhood $U$ of $p$, there is a positive integer $n$ such that there is only one set in $G_{n}$ containing $p$ and it is a subset of $U$.

Proof. That a space with such a sequence is developable is trivial. Suppose $S$ is developable. By Theorem 4 of [16], there is a sequence $H_{1}, H_{2}, \ldots$ of open collections (not covers) such that if $p$ is a point and $U$ is an open set containing $p$, there is a positive integer $n$ such that $\left\{V \in H_{n}: p \in V\right\}$ is a nonempty finite set containing a subset of $U$. For each $n$ and $k$, let $H_{n k}=\{V: V$ is the intersection of exactly $k$ members of $\left.H_{n}\right\}$. (This technique may be found in [13].) Suppose $p$ is a point and $U$ is an open set containing $p$. There exist $n$ and $k$ such that $H_{n}$ has exactly $k$ members containing $p$ and one of them is a subset of $U . H_{n k}$ has only one member containing $p$ and it is a subset of $U$.

Since every closed set in a developable space is a $G_{\delta}$, for each $n$ and $k$ there exists a sequence $\left\langle V_{n k i}: i \in N\right\rangle$ such that $\bigcap_{i} V_{n k i}=S-$ $\cup H_{n k}$. Let $H_{n k i}=H_{n k} \cup\left\{V_{n k i}\right\}$. The sequence $H_{n k i}$ is the desired development.

4.2. The technique used in 4.1 can be used to obtain a simpler proof of Theorem 4 of [16] which does not depend on the lemma stated there.

\section{REFERENCES}

1. O. H. Alzoobaee, Completion of Moore spaces, Thesis, University of Iowa, 1962.

2. S. Armentrout, Completing Moore spaces, Proc. Top. Conf. Arizona State University, (1967), 22-35.

3. G. D. Creede, Embedding of complete Moore spaces, Proc. Amer. Math. Soc., 28 (1971), 609-612.

4. P. Fletcher and W. F. Lindgren, Orthocompactness and strong Čech completeness in Moore spaces, Duke Math. J., 39 (1972), 753-766.

5. J. W. Green, Moore-closed spaces, completeness and centered bases, Gen. Top. and its Appl., 4 (1974), 297-313.

6. - Filters and $C$ - and $C^{*-e m b e d d i n g s, ~ G e n . ~ T o p . ~ a n d ~ i t s ~ A p p l ., ~} 3$ (1973), 97109.

7. R. L. Moore, Foundations of point set theory, Amer. Math. Soc. Colloq. Pub., Vol. 13, rev. ed., 1962.

8. A. D. My̌skis, On the concept of boundary, Mat. Sb. N. S., 25 (67), 387-414 (1949)= Amer. Math. Soc. Transl., (1) No. 51 (1951), 3-38.

9. C. Pixley and P. Roy, Uncompletable Moore spaces, Proc. Top. Conf. Auburn Univ., (1969), 75-85.

10. G. M. Reed, On screenability and metrizability of Moore spaces, Canad. J. Math., 23 (1971), 1087-1092.

11. J. N. Reed, On complete and semicomplete first countable spaces, Proc. Top. Conf. Univ. Oklahoma, (1972), 253-256. 
12. J. H. Roberts, A property related to completeness, Bull. Amer. Math. Soc., 38 (1932), 835-838.

13. D. Rolfsen, Alternative metrization proofs, Canad. J. Math., 18 (1966), 750-757.

14. M. E. Rudin, Concerning abstract spaces, Duke Math. J., 17 (1950), 317-327.

15. K. E. Whipple, Cauchy sequences in Moore spaces, Pacific J. Math., 18 (1966), 191-199.

16. J. M. Worrell, Jr. and H. H. Wicke, Characterizations of developable topological spaces, Canad. J. Math., 17 (1965), 820-830.

17. L. Zippen, On a problem of $N$. Aronszajn and an axiom of $R$. L. Moore, Bull. Amer. Math. Soc., 37 (1931), 276-280.

Received March 26, 1973.

The University of OKLahoma 



\title{
PACIFIC JOURNAL OF MATHEMATICS
}

\author{
EDITORS
}

RICHARD ARENS (Managing Editor)

University of California

Los Angeles, California 90024

R. A. Beaumont

University of Washington

Seattle, Washington 98105
J. DugundJI

Department of Mathematics University of Southern California Los Angeles, California 90007

D. Gilbarg and J. Milgram

Stanford University

Stanford, California 94305

\section{ASSOCIATE EDITORS}

E. F. BECKENBACH

B. H. NeUManN

F. WOLF

K. YosHIDA

\section{SUPPORTING INSTITUTIONS}

UNIVERSITY OF BRITISH COLUMBIA

GALIFORNIA INSTITUTE OF TECHNOLOGY

UNIVERSITY OF CALIFORNIA

MONTANA STATE UNIVERSITY

UNIVERSITY OF NEVADA

NEW MEXICO STATE UNIVERSITY

OREGON STATE UNIVERSITY

UNIVERSITY OF OREGON

OSAKA UNIVERSITY
UNIVERSITY OF SOUTHERN CALIFORNIA

STANFORD UNIVERSITY

UNIVERSITY OF TOKYO

UNIVERSITY OF UTAH

WASHINGTON STATE UNIVERSITY

UNIVERSITY OF WASHINGTON

AMERICAN MATHEMATICAL SOCIETY NAVAL WEAPONS CENTER 


\section{Pacific Journal of Mathematics}

\section{Vol. 57, No. $1 \quad$ January, 1975}

Keith Roy Allen, Dendritic compactification ......................... 1

Daniel D. Anderson, The Krull intersection theorem ..................... 11

George Phillip Barker and David Hilding Carlson, Cones of diagonally dominant

matrices .............................................

David Wilmot Barnette, Generalized combinatorial cells and facet splitting ........

Stefan Bergman, Bounds for distortion in pseudoconformal mappings ............

Nguyên Phuong Các, On bounded solutions of a strongly nonlinear elliptic

equation ...............................................

Philip Throop Church and James Timourian, Maps with 0-dimensional critical

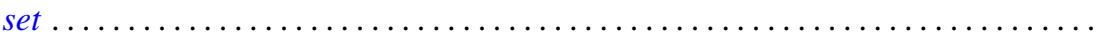

G. Coquet and J. C. Dupin, Sur les convexes ubiquitaires ................. 67

Kandiah Dayanithy, On perturbation of differential operators............... 85

Thomas P. Dence, A Lebesgue decomposition for vector valued additive set

functions .............................................. 91

John Riley Durbin, On locally compact wreath products.................. 99

Allan L. Edelson, The converse to a theorem of Conner and Floyd ............. 109

William Alan Feldman and James Franklin Porter, Compact convergence and the

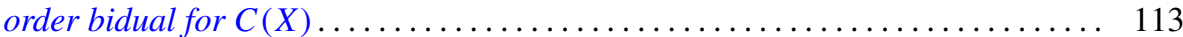

Ralph S. Freese, Ideal lattices of lattices......................... 125

R. Gow, Groups whose irreducible character degrees are ordered by divisibility ... 135

David G. Green, The lattice of congruences on an inverse semigroup ............ 141

John William Green, Completion and semicompletion of Moore spaces .......... 153

David James Hallenbeck, Convex hulls and extreme points of families of starlike and close-to-convex mappings .................................. 167

Israel (Yitzchak) Nathan Herstein, On a theorem of Brauer-Cartan-Hua type ...... 177

Virgil Dwight House, Jr., Countable products of generalized countably compact spaces ............................................ 183

John Sollion Hsia, Spinor norms of local integral rotations. I . . . . . . ......... 199

Hugo Junghenn, Almost periodic compactifications of transformation

semigroups....................................

Shin'ichi Kinoshita, On elementary ideals of projective planes in the 4-sphere and oriented $\Theta$-curves in the 3 -sphere ............................ 217

Ronald Fred Levy, Showering spaces............................ 223

Geoffrey Mason, Two theorems on groups of characteristic 2-type............. 233

Cyril Nasim, An inversion formula for Hankel transform ................... 255

W. P. Novinger, Real parts of uniform algebras on the circle ................ 259

T. Parthasarathy and T. E. S. Raghavan, Equilibria of continuous two-person

games............................................... 265

John Pfaltzgraff and Ted Joe Suffridge, Close-to-starlike holomorphic functions of several variables ....................................... 271

Esther Portnoy, Developable surfaces in hyperbolic space .................. 281

Maxwell Alexander Rosenlicht, Differential extension fields of exponential type . . . 289

Keith William Schrader and James Lewis Thornburg, Sufficient conditions for the

existence of convergent subsequences ........................... 301

Joseph M. Weinstein, Reconstructing colored graphs .................. 307 